\title{
Upregulation of Long Non-Coding RNA Small Nucleolar RNA Host Gene 12 Contributes to Cell Growth and Invasion in Cervical Cancer by Acting as a Sponge for MiR-424-5p
}

\author{
Jing Dong ${ }^{\mathrm{a}}$ Qing Wanga Li Li $^{\mathrm{b}} \quad$ Xiao-jin Zhang ${ }^{\mathrm{a}}$
}

${ }^{a}$ Center of Diagnosis and Treatment for Cervical Diseases, Obstetrics and Gynecology Hospital of Fudan University, Shanghai, ' Department of Obstetrics and Gynecology, Jiangyin Third people's Hospital, Jiangyin, Jiangsu, China

\section{Key Words}

Snhg12 • Cervical cancer $\cdot$ MiR-424-5p • Proliferation • Apoptosis

\begin{abstract}
Background/Aims: Cervical cancer, which is one of the most aggressive cancers affecting females, has high rates of recurrence and mortality. Small nucleolar RNA host gene 12 (SNHG12) is known to promote the progression of several cancers; however, its exact effects and molecular mechanisms in cervical cancer remain unknown. Methods: Real-time quantitative PCR was used to determine the expression level of SNHG12 in cervical cancer tissues and cell lines. Loss-of-function assays were performed to examine the effect of SNHG12 on the proliferation, apoptosis, migration and invasion of cervical cancer cells in vitro and tumor growth in vivo. Luciferase experiments were employed to explore the interactions between SNHG12 and miR-424-5p. Results: SNHG12 was found to be abnormally elevated in human cervical cancer tissues compared with paired adjacent normal tissues. Moreover, high SNHG12 expression in tumor tissues was significantly correlated with vascular involvement, lymph node metastasis, advanced FIGO stage and poor prognosis. Furthermore, the knockdown of SNHG12 was found to inhibit proliferation, migration and invasion of cervical cancer cells in vitro, and silencing SNHG12 was shown to suppress tumor growth in a nude mouse model. Mechanistic studies showed that SNHG12 functioned as an endogenous sponge for miR-424$5 p$, thereby downregulating the expression of miR-424-5p in cervical cancer. Furthermore, the inhibition of miR-424-5p in SNHG12-depleted cells partially reversed the effects on cervical cancer cell apoptosis, adhesion and invasion. Conclusion: In summary, our findings suggest that the tumor-promoting role of SNHG12 is to function as a molecular sponge, which negatively regulates miR-424-5p. These findings may provide a potent therapeutic target for cervical cancer.

J. Dong and Q. Wang contributed equally to this work.




\section{Cellular Physiology Cell Physiol Biochem 2018;45:2086-2094 \begin{tabular}{l|l} 
and Biochemistry Published onlıne: March 12, 2018 & $\begin{array}{l}\text { (c) } 2018 \text { The Author(s). Published by S. Karger AG, Basel } \\
\text { www.karger.com/cpb }\end{array}$
\end{tabular}}

\section{Introduction}

Cervical cancer is the fourth most common cancer and one of the most common malignant gynecologic cancers among women worldwide [1]. A growing body of evidence has indicated that both human papillomavirus (HPV) and aberrant gene expression contribute to cervical cancer development and precancerous lesions $[2,3]$. Despite the advances that have been achieved in therapeutic strategies including surgery and resection, either alone or in combination with radiotherapy and chemotherapy, the five-year overall survival rate remains poor because most cervical cancer patients are diagnosed at an advanced stage, with high rates of lymphatic metastasis and distant metastases [4-6]. Therefore, it is necessary to investigate the molecular and biological mechanisms that promote the progress of cervical tumors and to develop better therapeutic strategies to treat cervical cancer.

Long non-coding RNAs (lncRNAs), which constitute a large proportion of the transcriptome, generate RNA transcripts greater than 200 nucleotides in length and play an importantrole in regulating gene expression for biological processesincluding embryogenesis, stem-cell biology and cellular differentiation $[7,8]$. As studies on lncRNAs have advanced, it has been recognized that IncRNAs act as tumor suppressor genes or oncogenes via mediating tumor cell proliferation, growth, apoptosis, invasion and metastasis [9-13]. For instance, the lncRNA metastasis-associated lung adenocarcinoma transcript 1 (MALAT1) was found to promote cell cycle progression and tumorigenesis through regulating E2F1 transcription factor activity [14]. The lncRNA nuclear enriched abundant transcript 1 (NEAT1) was found to be overexpressed and associated with tumor progression in prostate cancer [15]. Recent studies have demonstrated that the IncRNA small nucleolar RNA host gene 12 (SNHG12) was increased in several cancers, including osteosarcoma, breast cancer and hepatocellular carcinoma [16-18]. However, the expression and role of SNHG12 in cervical cancer remain unknown and warrant investigation.

In the current study, we explored the expression of SNHG12 and the relationship between SNHG12 and clinicopathological variables in cervical cancer. The regulatory mechanism and function of SNHG12 were further investigated, and our results showed that SNHG12 acted as a competitive endogenous RNA (ceRNA) for miR-424-5p, which is involved in cervical cancer progression.

\section{Materials and Methods}

\section{Tissue specimens and cell lines}

The paired cervical cancer tissues and the adjacent non-tumor tissues were collected following curative surgical resection in patients with HCC in the Obstetrics \& Gynecology Hospital of Fudan University between July 2008 and November 2010. Informed written consent for scientific use was obtained from each patient, and this study was approved and supervised by the Ethics Committee of Fudan University and conducted in accordance with the Helsinki Declaration. All samples were immediately snap-frozen in liquid nitrogen prior to total RNA extraction.

Five human cervical cancer cell lines (C33A, ME-180, CaSki, HeLa and SiHa) and one immortalized cervical epithelial cell line (NC104) were purchased from the Cell Center of Shanghai Institutes for Biological Sciences and cultured as described in our previous study [19].

Quantitative reverse transcription-polymerase chain reaction analysis

Total RNA was extracted from tissues and cultured cells using TRIzol reagent (Invitrogen, Carlsbad, CA) according to the manufacturer's instructions. Quantitative RT-PCR (qRT-PCR) was performed as previously described [19]. The following specific primers were used: GAPDH forward: 5' -TGACTTCAACAGCGACACCCA-3', GAPDH reverse: 5'-CACCCTGTTGCTGTAGCCAAA-3'; SNHG12 forward: 5'- TCTGGTGATCGAGGACTTCC-3', SNHG12 reverse: 5' - ACCTCCTCAGTATCACACACT-3'.

Immunohistochemistry (IHC) staining

IHC staining was performed using a Ki-67 (Abcam, CA, USA) antibody according to the previous report [20]. 


\section{Cellular Physiology Cell Physiol Biochem 2018;45:2086-2094 \begin{tabular}{l|l} 
and Biochemistry Published 10.1159/000488045 & $\begin{array}{l}\text { (c) 2018 The Author(s). Published by S. Karger AG, Basel } \\
\text { www.karger.com/cpb }\end{array}$
\end{tabular}}

Dong et al.: Lnc SNHG12 Acts as a Cerna for MiR-424-5p in Cervical Cancer

\section{Plasmids construction and luciferase reporter assay}

Two short hairpin RNAs targeting SNHG12 were designed and synthetized by Sangon (Shanghai, China). The short hairpin RNAs were subcloned into the pLKO.1-puro vector (Addgene, Cambridge, MA, USA). The lentivirus was produced by the co-transfection of constructs pCMV-dR8.2 and VSVG into HEK293T cells using Lipofectamine ${ }^{\mathrm{TM}} 2000$ (Life Technologies, Carlsbad, CA, USA). Cell supernatants were collected and filtered $48 \mathrm{~h}$ later. Cervical cancer cells were infected with the virus and selected with $2 \mu \mathrm{g} / \mathrm{mL}$ puromycin (Sigma-Aldrich, St. Louis, MO, USA). QRT-PCR was used to verify the knockdown efficiency of the stable knockdown cells.

The full-length SNHG12 sequence was amplified by PCR, and the PCR product was subcloned into the pMir-Reporter plasmid (Life Technologies) to produce the wild-type reporter (SNHG12-WT). For the mutant reporter (SNHG12-MUT) construct, site-directed mutagenesis of the miR-424-5p target site was performed using the Quick-change mutagenesis kit (Stratagene, Germany). The MiR-424-5p mimic and inhibitor were purchased from ThermoFisher. The Reporter plasmid and the miR-424-5p mimic were transfected into HEK293T cells using Lipofectamine ${ }^{\mathrm{TM}}$ 2000. Luciferase activity was measured using the Dual Luciferase Reporter Assay System (Promega, USA), according to the manufacturer's instructions, $24 \mathrm{~h}$ post-transfection.

\section{Cell proliferation and colony-forming assay}

For the proliferation assay, $2 \times 10^{3}$ cells were seeded into a 96-well plate, and $10 \mu \mathrm{L}$ of Cell Counting Kit-8 (Dojindo, Japan) was added to each well at various time points. The absorbance was detected at 450 nm using a microplate ELISA reader (Bio-Rad, Hercules, CA).

For the colony formation assay, 800 cells were seeded in a 6-well plate and cultured in complete culture medium; the medium was replaced every 3 days. Colonies containing at least 50 cells on the plates were fixed with 4\% polyoxymethylene and stained with 1\% crystal violet for 15 min 15 days later.

\section{Cell apoptosis analysis by flow cytometry}

Cells were harvested and washed twice with $1 \times$ PBS. Then, cells were stained with Annexin-V and PI (BD Biosciences, Franklin Lakes, NJ, USA) supplemented with $1 \mu \mathrm{g} / \mathrm{ml}$ RNase (Sigma-Aldrich) at $37^{\circ} \mathrm{C}$ in the dark for $30 \mathrm{~min}$. The percentage of cells undergoing apoptosis was analyzed on a FACScalibur flow cytometer using CellQuestPro software (Becton Dickinson, USA).

\section{Cell migration and invasion assays}

Cell migratory and invasive abilities were assessed using a transwell chamber with 8 - $\mu \mathrm{m}$ pores (Costar, Corning, NY, USA), according to the manufacturer's instruction. For the migration assay, $4 \times 10^{5}$ cells in 200 $\mu \mathrm{L}$ of serum-free medium were added to the upper chamber. A total of $600 \mu \mathrm{L}$ of compete medium was added to the lower chamber as a chemoattractant. Following $24 \mathrm{~h}$ of incubation, the migratory cells were fixed and stained with a $1 \%$ crystal violet solution, and then the cells were photographed under a confocal microscope.

\section{In vivo experiment}

Four-week-old female nude mice were purchased from the Shanghai LAC Laboratory Animal Co. Ltd. (Shanghai, China). Then, $1 \times 10^{7}$ cells transduced with either control or SNHG12 shRNA in $100 \mu \mathrm{L}$ of serumfree medium were injected into the left flank of each mouse (8 mice per group). Five weeks after receiving the injections, the mice were sacrificed and the tumors were excised and photographed. The tumor volume (in $\mathrm{mm}^{3}$ ) was examined by caliper measurements every week and calculated using the following formula: volume $=$ length $\times$ width $^{2} / 2$. The tumors were then weighed and fixed for IHC staining. This study was performed in accordance with the Care and Use of Laboratory Animals of the National Institutes of Health.

\section{Statistical analysis}

All of the results are presented as the mean \pm standard deviation from three independent experiments. The Student's t-test was used to evaluate significant differences between the two groups. The relationship between SNHG12 expression and clinicopathological characteristics was evaluated using the Chi-square test. The Pearson's correlation analysis was performed to assess the correlation between SNHG12 and miR424-5p expression. $\mathrm{P}<0.05$ was considered to be statistically significant. 


\section{Cellular Physiology Cell Physiol Biochem 2018;45:2086-2094

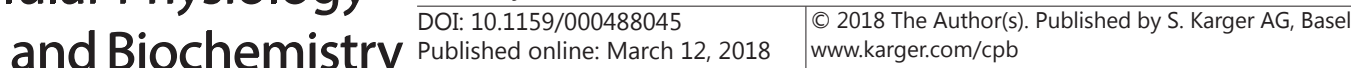

\section{Results}

\section{Aberrant SNHG12 expression in paired cervical cancer tissues and cell lines}

To explore the role of SNHG12 in cervical cancer, the expression SNHG12 was examined in cervical cancer tissue samples and adjacent normal tissue by qRT-PCR. Our results showed that SNHG12 expression was significantly upregulated in cervical cancer tissues compared to the corresponding normal tissues (Fig. 1A and B). Furthermore, SNHG12 expression was significantly higher in FIGO stage II than FIGO stage I (Fig. 1C). In addition, the relative expression levels of SNHG12 in the cervical cancer cell lines were significantly increased compared with the immortalized cervical epithelial cell line (NC104) (Fig. 1D). These data revealed that SNHG12 was abnormally elevated in cervical cancer and therefore may function as an oncogene.

\section{Correlations between SNHG12 and clinicopathological parameters in cervical cancer}

The association between SNHG12 expression and clinicopathological features is presented in Table 1. Our results showed that the upregulation of SNHG12 was significantly associated with FIGO stage $(P=0.0272)$ and lymph node metastasis $(P=0.004)$. However, there were no significant associations between SNHG12 expression and the other clinicopathological features. To evaluate the relationship between SNHG12 expression and the outcome of patients with cervical cancer, the patients were divided into either high SNHG12 expression or low SNHG12 expression, according to the mean value of SNHG12 expression measured for each patient. The Kaplan-Meier survival curve indicated that the overall survival rate was lower in the high SNHG12 expression group than in the low SNHG12 expression group (Fig. 1E). These data suggested that SNHG12 may be involved in the progression and development of cervical cancer.

\section{Knockdown of SNHG12 inhibits cell proliferation}

Since SNHG12 expression was found to be upregulated in cervical cancer, we further explored the biological functions of SNHG12 in cervical cancer cells by knocking down the expression of SNHG12 in SiHa and CaSki cells, which exhibited the highest endogenous levels of SNHG12 expression. The QRT-PCR results confirmed that SNHG12 was remarkably decreased as a result of the two short hairpin RNAs (Fig. 2A). The proliferation ability of SiHa and CaSki cells decreased after SNHG12 was silenced, according to the CCK-8 assays (Fig. 2B). We selected shRNA\#2 for further experiments, due to its superior inhibition efficiency. Furthermore, colony forming assays also indicated that the knockdown of SNHG12 markedly decreased the number and size of the colonies (Fig. 2C). We also

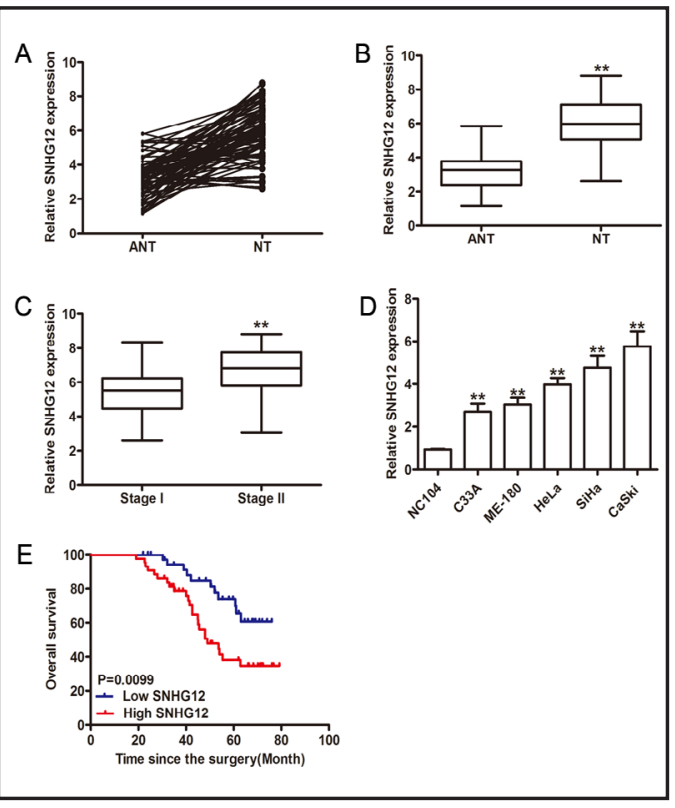

Fig. 1. LncRNA SNHG12 was significantly increased in cervical cancer tissues and cell lines and was correlated with poor prognosis. (A) The expression level of HOXA-AS2 was detected in human breast cancer tissues and matched adjacent normal tissues by qRT-PCR. (B) Upregulation of SNHG12 in cervical cancer tissues compared with matched adjacent normal tissues. (C) Expression of SNHG12 is increased in FIGO stage II relative to FIGO stage I. (D) SNHG12 expression in cervical cancer cell lines (C33A, ME-180, CaSki, HeLa and SiHa) and an immortalized cervical epithelial cell line (NC104). (E) Kaplan-Meier curve of overall survival rate of cervical cancer patients with high SNHG12 levels ( $\mathrm{n}=$ 38) and low SNHG12 levels $(\mathrm{n}=38) .{ }^{* *} \mathrm{P}<0.01$. 
explored whether SNHG12 promoted the proliferation of cervical cancer cells via influencing apoptosis. Flow cytometry revealed that depletion of SNHG12 induced cervical cancer cell apoptosis (Fig. 2D). These findings indicated that the downregulation of SNHG12 inhibited cell proliferation via regulating cell apoptosis in vitro.

Attenuated expression of SNHG12 inhibits cervical cancer cell migration and invasion

Previous studies have found that SNHG12 expression was increased in and contribute to the metastasis of several types of cancer [16-18]. We assessed the effect of SNHG12 on cervical cancer cells, and we observed that the downregulation of SNHG12 led to a significant decrease in the migratory and invasive capacities of the cells (Fig. 3A and $B$ ). These findings suggested that the downregulation of SNHG12 attenuated the migration and invasion abilities of cervical cancer cells.

SNHG12 silencing suppresses tumorigenesis in nude mice

Based upon the observed inhi-

Table 1. Association between SNHG12 expression and clinicopathological variables of cervical cancer patients

\begin{tabular}{|c|c|c|c|c|}
\hline \multirow{2}{*}{ Clinicopathological Variable } & \multirow{2}{*}{ Total $(\mathrm{n}=81)$} & \multicolumn{2}{|c|}{ FOXC1 expression } & \multirow[t]{2}{*}{$P$ value } \\
\hline & & high $(n=43)$ & low $(n=38)$ & \\
\hline Age, years & & & & 0.6139 \\
\hline$\leq 45$ & 25 & 15 & 10 & \\
\hline$>45$ & 56 & 38 & 18 & \\
\hline FIGO stage & & & & 0.0272 \\
\hline I & 46 & 16 & 30 & \\
\hline II & 35 & 21 & 14 & \\
\hline Differentiation grade & & & & 0.5016 \\
\hline Well/Moderate & 48 & 22 & 26 & \\
\hline Poor & 33 & 18 & 15 & \\
\hline Carcinoma diameter $(\mathrm{cm})$ & & & & 0.3692 \\
\hline$\leq 4$ & 50 & 28 & 22 & \\
\hline$>4$ & 31 & 14 & 17 & \\
\hline Pathologic types & & & & 1 \\
\hline Squamous cell carcinoma & 51 & 25 & 26 & \\
\hline Adenocarcinoma & 30 & 14 & 16 & \\
\hline Lymph node metastasis & & & & 0.004 \\
\hline No & 52 & 14 & 38 & \\
\hline Yes & 29 & 18 & 11 & \\
\hline Myometrium invasion & & & & 0.8255 \\
\hline$<1 / 2$ & 38 & 20 & 18 & \\
\hline$\geq 1 / 2$ & 43 & 24 & 19 & \\
\hline Vascular involvement & & & & 0.099 \\
\hline No & 58 & 45 & 13 & \\
\hline Yes & 23 & 13 & 10 & \\
\hline Stromal invasion & & & & 0.7738 \\
\hline$<66 \%$ & 61 & 36 & 25 & \\
\hline$\geq 66 \%$ & 15 & 8 & 7 & \\
\hline Vaginal involvement & & & & 0.7677 \\
\hline No & 14 & 7 & 7 & \\
\hline Yes & 67 & 39 & 28 & \\
\hline Parametrial infiltration & & & & 0.518 \\
\hline No & 70 & 29 & 41 & \\
\hline Yes & 11 & 6 & 5 & \\
\hline
\end{tabular}
bition of the proliferation of cervical cancer cells resulting from the knockdown of SNHG12 expression in vitro, a tumorigenesis assay in female nude mice was performed to verify whether silencing SNHG12 expression inhibited tumor growth in vivo. As shown in Fig. 4A, the tumor volume was smaller in the mice injected with CaSki cells transduced with SNHG12 shRNA than in the mice injected with the NC. The average weight of the tumors derived from SNHG12 knockdown CaSki cells was less than that of tumors derived from the NC (Fig. 4B). Depletion of SNHG12 in CaSki cells slowed the tumor growth curves (Fig. 4C). Furthermore, the expression of SNHG12 and miR-424-5p from xenograft tumors was measured by qRT-PCR, and our results indicated that SNHG12 was decreased but miR-424-

Fig. 2. Effect of SNHG12 in regulating cervical cancer cell proliferation. (A) qRT-PCR analysis of SNHG12 expression in CaSki and SiHa cells transduced with the negative control (SCR) or SNHG12 shRNAs. (B) Growth curves (C) colony-formation assay and (D) cell apoptosis of CaSki and SiHa cells transduced with the negative control (SCR) or SNHG12 shRNAs, analyzed by CCK-8 assay. ** $\mathrm{P}<0.01$.
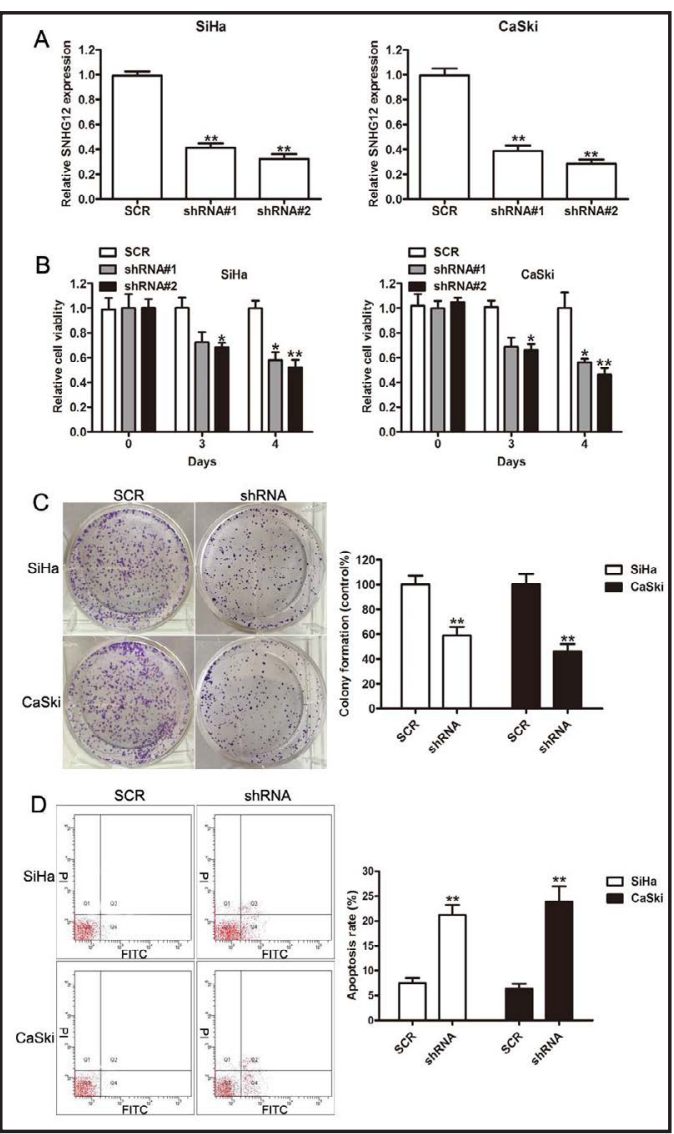

\section{KARGER}




\section{Cellular Physiology Cell Physiol Biochem 2018;45:2086-2094

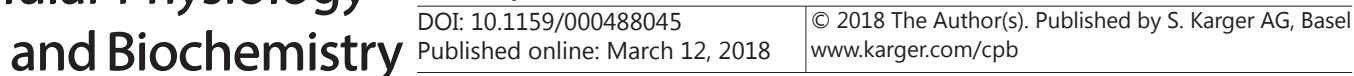 \\ Dong et al.: Lnc SNHG12 Acts as a Cerna for MiR-424-5p in Cervical Cancer}

$5 p$ was increased in the tumors of the SNHG12 shRNA group, compared to the SCR group (Fig. 4D and E). In addition, the proliferative activity of the tumor cells was assessed via staining for Ki67, which is a proliferation marker. Low Ki-67 expression was observed in the SNHG12 shRNA group (Fig. 4F). Therefore, our results demonstrated that the downregulation of SNHG12 could inhibit the proliferation of cervical cancer cells in vivo, which was consistent with the findings of the in vitro assays.

SNHG12 serves as an endogenous sponge for miR-424-5p in cervical cancer

Accumulating evidence has shown that IncRNAs can affect physiological and pathological processes through partly binding with miRNAs. The program StarBase v2.0 (http://starbase. sysu.edu.cn/) was used to predict potential lncRNA-miRNA interactions, and we found that SNHG12 has a putative binding site for the seed sequence of miR424-5p (Fig. 5A). Interestingly, miR-424 was found to be decreased in cervical cancer tissues, and the overexpression of miR-424 inhibited cell growth, migration and invasion $[21,22]$. To verify the interaction between SNHG12 and miR424-5p, a luciferase reporter assay was performed. The results indicated that miR-424-5p could significantly inhibit the reporter activities of SNHG12-WT but not SNHG12-MUT (Fig. 5B). MiR424-5p was increased in $\mathrm{SiHa}$ and CaSki cells after the knockdown of SNHG12 expression (Fig. 5C). Furthermore, we detected the expression of miR-424-5p in cervical cancer tissues by qRT-PCR. As shown in Fig. 5D, miR-424-5p was significantly downregulated in cervical cancer tissues, compared with the corresponding normal tissues. In addition, we identified a negative correlation between SNHG12 expression and miR-424-5p expression by the Pearson correlation test in patients with cervical cancer (Fig. 5E). These data suggested that SNHG12 promotes cell growth and mobility by negatively modulating miR424 expression.

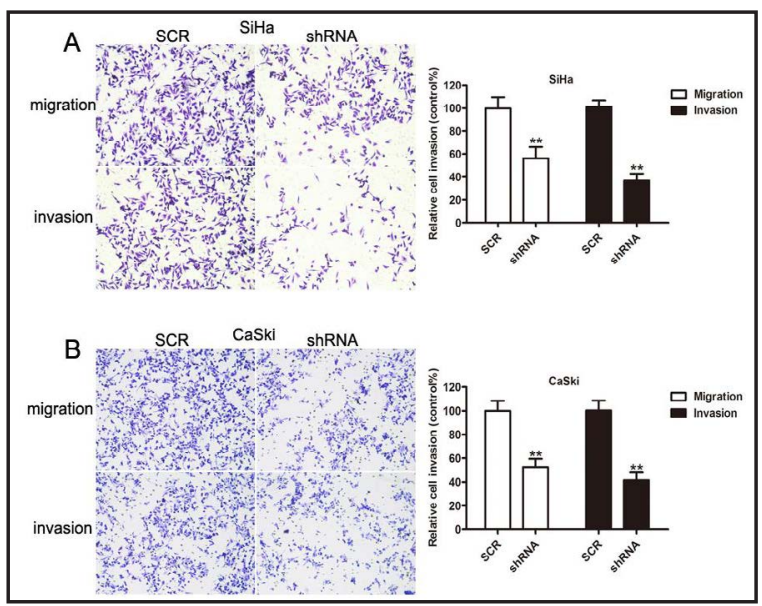

Fig. 3. Silencing of SNHG12 expression inhibits migration and invasion of cervical cancer cells. (A) Migratory and invasive capacities of SiHa cells were detected by transwell assays. (B) Migratory and invasive capacities of CaSki cells were assessed by transwell assays. ${ }^{* *} \mathrm{P}<0.01$.

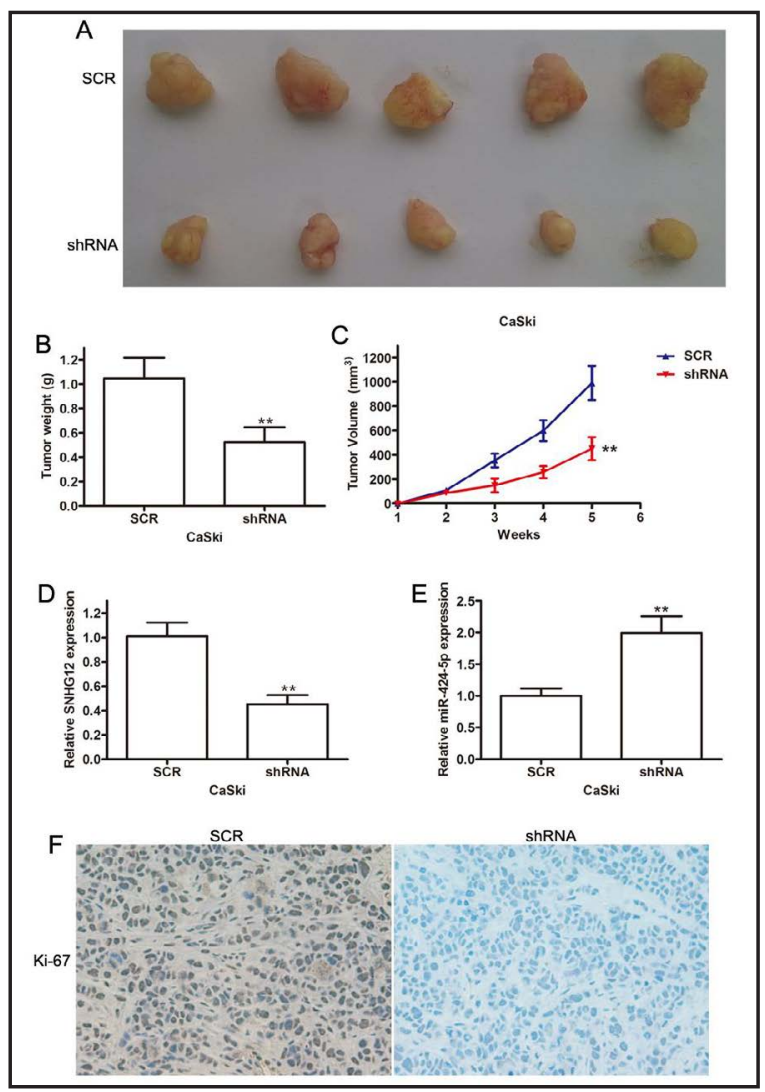

Fig. 4. Effects of SNHG12 on tumor growth in vivo. (A) Representative images of the injected tumors from the nude mice for tumorigenesis. (B) Quantitative analysis of the mean tumor weights. (C) Tumor volumes were measured at the indicated times. (D and E) qRT-PCR analysis of the expression levels of SNHG12 and miR-424-5p in the tumors from the xenograft tumor. (F) Representative IHC staining for Ki-67 in the tumors from the injected mice. ${ }^{* *} \mathrm{p}<0.01$. 
Fig. 5. miR-424-5p was directly regulated by SNHG12 in cervical cancer. (A) Schematic description of the hypothetical duplex formed by the interaction between the binding site in miR-424-5p and SNHG12 by StarBase v2.0. (B) The relative luciferase activities of the wild type or mutant SNHG12 reporter plasmid transfected with miR-424-5p in HEK393T. (C) qRT-PCR analysis of miR-424-5p expression in CaSki and SiHa cells following SNHG12 silencing. (D) qRT-PCR analysis of SNHG12 expression in cervical cancer tissues. (E) The relationship between SNHG12 and miR-424-

$5 \mathrm{p}$ was assessed by the Pearson's correlation analysis. ${ }^{* *} \mathrm{p}<0.01$.

Silencing miR-424-5p partially reversed the effects of SNHG12 knockdown in cervical cancer cells

To obtain direct evidence that the downregulation of miR-424$5 p$ was required for the effects of SNHG12, we transfected SNHG12depleted SiHa and CaSki cells with a miR-424-5p inhibitor, and the results confirmed that miR-424-5p was markedly suppressed (Fig. 6A). As expected, flow cytometric analysis indicated that reduced miR-424-5p expression decreased the apoptosis level of SNHG12-depleted cervical cancer cells compared to the control group (Fig. 6B). A transwell migration assay also demonstrated that silencing miR-424-5p markedly promoted the migratory and invasive abilities of SNHG12-depleted SiHa and CaSki cells (Fig. 6C and D). Taken together, these results suggested that miR-424-5p was involved in the SNHG12-mediated oncogenic activity.

\section{Discussion}

Emerging evidence has indicated that IncRNAs play versatile roles in many biological processes [23, 24].

Studies have shown that aberrant lncRNA expression influences cancer progression and can predict patient outcome [12,24]. In the present study, we examined the expression of SNHG12 in cervical cancer tissues, compared to the corresponding normal tissues, and we found that SNHG12 was significantly upregulated in cervical cancer tissue and that high SNHG12 was positively associated with FIGO stage, lymph node metastasis and a poor prognosis. The stable knockdown of SNHG12 inhibited the proliferation, migration and invasion of cervical cancer cells and promoted apoptosis. In addition, the knockdown of SNHG12 expression led
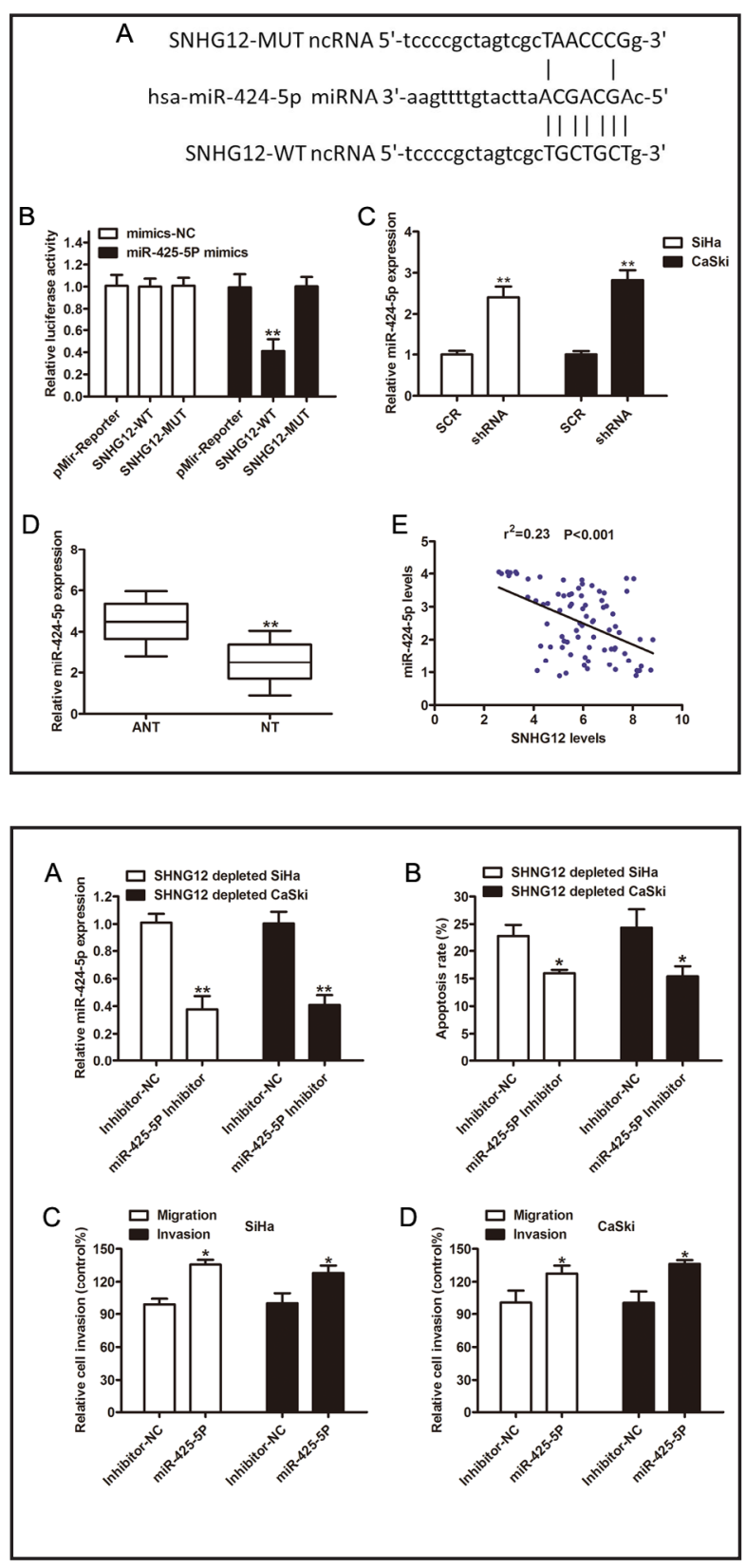

Fig. 6. SNHG12 regulated cell apoptosis and invasion by suppressing miR-424-5p in cervical cancer cells. (A) qRT-PCR was performed to determine the expression of miR-424-5p. (B) Cell apoptosis, (C and D) migratory and invasive capacities of SNHG12-depleted CaSki and SiHa cells were assessed. ${ }^{* *} \mathrm{p}<0.01$. 


\section{Cellular Physiology Cell Physiol Biochem 2018;45:2086-2094

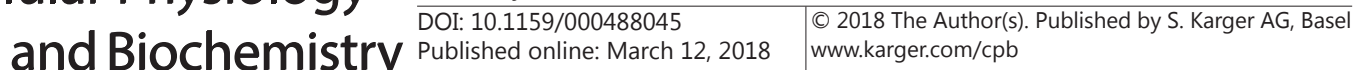

to a decrease in tumorigenic properties. Our results suggest that SNHG12 acts as an oncogene in cervical cancer pathogenesis and development.

SNHG12 was first identified in endometrial cancer in a microarray expression profile, and in that study, SNHG12 depletion partly repressed cell proliferation and induced apoptosis [25]. Further studies have shown that SNHG12 is significantly increased in hepatocellular carcinoma and is associated with tumor size, vascular invasion and TNM stage [18]. In accordance with the abovementioned findings, we observed that SNHG12 was significantly upregulated in cervical cancer tissues and in cervical cancer cell lines.

To further investigate the biological functions of SNHG12, shRNA-mediated gene silencing was used to explore the effects of SNHG12 on malignant features. Functional assays revealed that the inhibition of SNHG12 expression decreased cell proliferation both in vitro and in vivo, indicating that SNHG12 plays a critical role in the progression of cervical cancer. To explore the potential mechanism underlying the effects of SNHG12, an FCM assay determined that silencing SNHG12 expression promoted apoptosis and inhibited migration and invasion of cervical cancer cells. Our results confirmed that the overexpression of SNHG12 promoted cell proliferation and migration and inhibited apoptosis [16-18]. Our study expanded upon the current knowledge of the downstream regulatory mechanism of SNHG12. Bioinformatic prediction and dual-luciferase reporter assays revealed that SNHG12 functioned as a sponge for miR-424-5p, a known tumor suppressor, to promote cervical cancer development. We also found that miR-424-5p was downregulated in cervical cancer tissues, and, moreover, there was a negative correlation between SNHG12 and miR-424-5p expression in cervical cancer tissues. We then investigated whether miR-424-5p was involved in SNHG12-mediated oncogenic activity. Our results showed that silencing miR-424-5p in SNHG12-depleted cervical cancer cells reversed the effects on cell apoptosis, migration and invasion induced by the knockdown of SNHG12.

\section{Conclusion}

Our study showed that SNHG12 is upregulated in cervical tissues and cells, and high SNHG12 expression is associated with a poor prognosis in patients with cervical cancer. The knockdown of SNHG12 expression suppressed cell proliferation and invasion by modulating miR-424-5p expression. Nevertheless, the specific molecular mechanisms and other potential targets warrant further investigation.

\section{Acknowledgements}

This work was supported by the National Natural Science Foundation of China (NO. 81373869 and No. 30873351).

\section{Disclosure Statement}

The authors confirm that there is no conflict of interest.

\section{References}

1 Jemal A, Bray F, Center MM, Ferlay J, Ward E, Forman D: Global cancer statistics. CA Cancer J Clin 2011;61:69-90.

$\checkmark 2$ Subramanya D, Grivas PD: HPV and cervical cancer: Updates on an established relationship. Postgrad Med 2008;120:7-13.

-3 Schiffman M, Castle PE, Jeronimo J, Rodriguez AC, Wacholder S: Human papillomavirus and cervical cancer. Lancet 2007;370:890-907. 


\section{Cellular Physiology Cell Physiol Biochem 2018;45:2086-2094

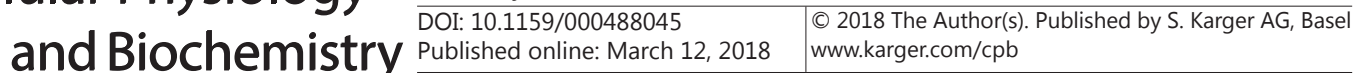

Dong et al.: Lnc SNHG12 Acts as a Cerna for MiR-424-5p in Cervical Cancer

4 Kilic S, Cracchiolo B, Gabel M, Haffty B, Mahmoud O: The relevance of molecular biomarkers in cervical cancer patients treated with radiotherapy. Ann Transl Med 2015;3:261.

5 Yin M, Zhang H, Li H, Li X, Liu Y, Chen X, Lou G, Li K: The toxicity and long-term efficacy of nedaplatin and paclitaxel treatment as neoadjuvant chemotherapy for locally advanced cervical cancer. J Surg Oncol 2012;105:206-211.

6 Hu T, Li S, Chen Y, Shen J, Li X, Huang K, Yang R, Wu L, Chen Z, Jia Y, Wang S, Cheng X, Han X, Lin Z, Xing H, Qu P, Cai H, Song X, Tian X, Xu H, Xu J, Zhang Q Xi L, Deng D, Wang H, Wang S, Lv W, Wang C, Xie X, Ma D: Matched-case comparison of neoadjuvant chemotherapy in patients with FIGO stage IB1-IIB cervical cancer to establish selection criteria. Eur J Cancer 2012;48:2353-2360.

7 Schmitz SU, Grote P, Herrmann BG: Mechanisms of long noncoding RNA function in development and disease. Cell Mol Life Sci 2016;73:2491-2509.

-8 Yin QF, Yang L, Zhang Y, Xiang JF, Wu YW, Carmichael GG, Chen LL: Long noncoding RNAs with snoRNA ends. Mol Cell 2012;48:219-230.

9 Chen X, Lun L, Hou H, Tian R, Zhang H, Zhang Y: The value of IncRNA HULC as a prognostic factor for survival of cancer outcome: A Meta-Analysis. Cell Physiol Biochem 2017;41:1424-1434.

10 Liu YY, Chen ZH, Peng JJ, Wu JL, Yuan YJ, Zhai ET, Cai SR, He YL, Song W: Up-regulation of long non-coding RNA XLOC_010235 regulates epithelial-to-mesenchymal transition to promote metastasis by associating with Snail1 in gastric cancer. Sci Rep 2017;7:2461.

11 Jiang R, Tang J, Chen Y, Deng L, Ji J, Xie Y, Wang K, Jia W, Chu WM, Sun B: The long noncoding RNA lnc-EGFR stimulates T-regulatory cells differentiation thus promoting hepatocellular carcinoma immune evasion. Nat Commun 2017;8:15129.

12 Lu X, Zhou C, Li R, Deng Y, Zhao L, Zhai W: Long noncoding RNA AFAP1-AS1 promoted tumor growth and invasion in cholangiocarcinoma. Cell Physiol Biochem 2017;42:222-230.

-13 Schmitt AM, Chang HY: Long noncoding RNAs in cancer pathways. Cancer Cell 2016;29:452-463.

-14 Derrien T, Guigo R: [Long non-coding RNAs with enhancer-like function in human cells]. Med Sci (Paris) 2011;27:359-361.

15 Chakravarty D, Sboner A, Nair SS, Giannopoulou E, Li R, Hennig S, Mosquera JM, Pauwels J, Park K, Kossai M, MacDonald TY, Fontugne J, Erho N, Vergara IA, Ghadessi M, Davicioni E, Jenkins RB, Palanisamy N, Chen Z, Nakagawa S, Hirose T, Bander NH, Beltran H, Fox AH, Elemento O, Rubin MA: The oestrogen receptor alpharegulated IncRNA NEAT1 is a critical modulator of prostate cancer. Nat Commun 2014;5:5383.

16 Ruan W, Wang P, Feng S, Xue Y, Li Y: Long non-coding RNA small nucleolar RNA host gene 12 (SNHG12) promotes cell proliferation and migration by upregulating angiomotin gene expression in human osteosarcoma cells. Tumour Biol 2016;37:4065-4073.

17 Wang O, Yang F, Liu Y, Lv L, Ma R, Chen C, Wang J, Tan Q, Cheng Y, Xia E, Chen Y, Zhang X: C-MYC-induced upregulation of IncRNA SNHG12 regulates cell proliferation, apoptosis and migration in triple-negative breast cancer. Am J Transl Res 2017;9:533-545.

18 Lan T, Ma W, Hong Z, Wu L, Chen X, Yuan Y: Long non-coding RNA small nucleolar RNA host gene 12 (SNHG12) promotes tumorigenesis and metastasis by targeting miR-199a/b-5p in hepatocellular carcinoma. J Exp Clin Cancer Res 2017;36:11.

19 Dong J, Sui L, Wang Q, Chen M, Sun H: MicroRNA-26a inhibits cell proliferation and invasion of cervical cancer cells by targeting protein tyrosine phosphatase type IVA 1. Mol Med Rep 2014;10:1426-1432.

20 Li Y, Xu Y, Ye K, Wu N, Li J, Liu N, He M, Lu B, Zhou W, Hu R: Knockdown of tubulin polymerization promoting protein family member 3 suppresses proliferation and induces apoptosis in Non-Small-Cell lung cancer. J Cancer 2016;7:1189-1196.

-21 Xu J, Li Y, Wang F, Wang X, Cheng B, Ye F, Xie X, Zhou C, Lu W: Suppressed miR-424 expression via upregulation of target gene Chk1 contributes to the progression of cervical cancer. Oncogene 2013;32:976987.

22 Wang X, Li Q, Jin H, Zou H, Xia W, Dai N, Dai XY, Wang D, Xu CX, Qing Y: MiR-424 acts as a tumor radiosensitizer by targeting aprataxin in cervical cancer. Oncotarget 2016;7:77508-77515.

23 Kung JT, Colognori D, Lee JT: Long noncoding RNAs: Past, present, and future. Genetics 2013;193:651-669.

-24 Mercer TR, Dinger ME, Mattick JS: Long non-coding RNAs: Insights into functions. Nat Rev Genet 2009;10:155-159.

-25 Zhai W, Li X, Wu S, Zhang Y, Pang H, Chen W: Microarray expression profile of IncRNAs and the upregulated ASLNC04080 lncRNA in human endometrial carcinoma. Int J Oncol 2015;46:2125-2137. 